\title{
Ex Vivo Activity Quantification in Micrometastases at the Cellular Scale Using the $\alpha$-Camera Technique
}

\author{
Nicolas Chouin ${ }^{1,2}$, Sture Lindegren ${ }^{2}$, Sofia H.L. Frost ${ }^{2}$, Holger Jensen ${ }^{3}$, Per Albertsson ${ }^{4}$, Ragnar Hultborn ${ }^{4}$, Stig Palm$^{2}$, \\ Lars Jacobsson ${ }^{2}$, and Tom Bäck ${ }^{2}$ \\ ${ }^{1}$ LUNAM Université, Oniris, AMaROC, Nantes, France; ${ }^{2}$ Department of Radiation Physics, Institute of Clinical Sciences, \\ Sahlgrenska Academy, University of Gothenburg, Gothenburg, Sweden; ${ }^{3}$ Cyclotron and PET Unit, Rigshospitalet, Copenhagen, \\ Denmark; and ${ }^{4}$ Department of Oncology, Institute of Clinical Sciences, Sahlgrenska Academy, University of Gothenburg, Gothenburg, \\ Sweden
}

\begin{abstract}
Targeted $\alpha$-therapy (TAT) appears to be an ideal therapeutic technique for eliminating malignant circulating, minimal residual, or micrometastatic cells. These types of malignancies are typically infraclinical, complicating the evaluation of potential treatments. This study presents a method of ex vivo activity quantification with an $\alpha$-camera device, allowing measurement of the activity taken up by tumor cells in biologic structures a few tens of microns. Methods: We examined micrometastases from a murine model of ovarian carcinoma after injection of a radioimmunoconjugate labeled with ${ }^{211}$ At for TAT. At different time points, biologic samples were excised and cryosectioned. The activity level and the number of tumor cells were determined by combined information from 2 adjacent sections: one exposed to the $\alpha$-camera and the other stained with hematoxylin and eosin. The time-activity curves for tumor cell clusters, comprising fewer than 10 cells, were derived for 2 different injected activities (6 and $1 \mathrm{MBq})$. Results: High uptake and good retention of the radioimmunoconjugate were observed at the surface of tumor cells. Dosimetric calculations based on the measured time-integrated activity indicated that for an injected activity of $1 \mathrm{MBq}$, isolated tumor cells received at least 12 Gy. In larger micrometastases ( $\leq 100 \mu \mathrm{m}$ in diameter), the activity uptake per cell was lower, possibly because of hindered penetration of radiolabeled antibodies; however, the mean absorbed dose delivered to tumor cells was above $30 \mathrm{~Gy}$, due to cross-fire irradiation. Conclusion: Using the $\alpha$-camera, we developed a method of ex vivo activity quantification at the cellular scale, which was further applied to characterize the behavior of a radiolabeled antibody administered in vivo against ovarian carcinoma. This study demonstrated a reliable measurement of activity. This method of activity quantification, based on experimentally measured data, is expected to improve the relevance of small-scale dosimetry studies and thus to accelerate the optimization of TAT.
\end{abstract}

Key Words: ${ }^{211} \mathrm{At}$; radioimmunotherapy; quantification; small-scale dosimetry

J Nucl Med 2013; 54:1347-1353

DOI: 10.2967/jnumed.112.113001

\footnotetext{
Received Aug. 31, 2012; revision accepted Feb. 7, 2013.

For correspondence or reprints contact: Nicolas Chouin, AMaROC team, Oniris, Site de la Chantrerie-B.P. 40706, 44307 Nantes Cedex 03, France. E-mail: nicolas.chouin@oniris-nantes.fr

Published online Jun. 12, 2013.

COPYRIGHT (C) 2013 by the Society of Nuclear Medicine and Molecular Imaging, Inc.
}

O the basis of the characteristics of the $\alpha$-particle, targeted $\alpha$-therapy (TAT) has been acknowledged as a potentially efficient therapeutic technique (1) for decades. $\alpha$-particles have a short range in tissue, making the irradiation specific, and a high linear energy transfer, making them highly cytotoxic (2). These properties make them suitable for the treatment of hematologic malignancies or minimal residual or micrometastatic diseases $(3,4)$. The first clinical trials using TAT have been started for the treatment of leukemia (5,6), ovarian carcinoma (7), and residual tumor cells after surgery of malignant brain tumors (8).

Micrometastatic diseases are infraclinical and hardy or not localized with in vivo imaging techniques. Thus, targeted radionuclide therapies of tumor cells or micrometastases require complex evaluations. Activity quantification of micrometric lesions by imaging is difficult in patients because of the limited spatial resolution. Only rough estimations of the activity of isolated tumor cells are feasible, and they are based on assumptions of tumor burden size $(5,6)$.

Consequently, preclinical experiments are required to assess the targeting efficiency of the radiolabeled vector and to identify the optimal conditions for treatment delivery. The first method for selecting the most promising vector for delivering $\alpha$-emitters to tumors is based on in vitro measurements of the activity taken up by isolated cells (9-11). However, in vivo evaluations are complex, even in animal studies, for determining tumor uptake of radioimmunoconjugates in the context of circulating cells, residual cells, or minimal diseases. To perform accurate dosimetric studies and estimate the therapeutic window of a potential treatment, it is essential to measure the temporal distribution of activity within the micrometric lesions. To that purpose, small-scale ex vivo quantifications (i.e., assessment of the temporal distribution of the radionuclide, presumably presenting the number of radiolabeled vectors per tumor cell) can provide invaluable information.

Recently, a device based on autoradiography, the $\alpha$-camera, was developed and evaluated by our group (12). This device provides images of activity distribution (of $\alpha$-emitters) with good spatial resolution (around $35 \mu \mathrm{m}$ ). In the present study, we used the $\alpha$-camera to develop a method to quantify the activity taken up by small micrometastases excised from a murine model of ovarian carcinoma after TAT. We hypothesized that this method would allow estimations of relevant, small-scale, mean absorbed doses and thus provide a means to confirm the delivery of potentially therapeutic absorbed doses to tumor cells. 


\section{MATERIALS AND METHODS}

\section{Overview}

The general method of quantification is described in Figure 1. The samples comprised small micrometastases excised from a nude mouse model of ovarian carcinoma (OVCAR-3) after TAT. The method was developed to measure the tumor cell uptake of a monoclonal antibody (mAb), MX35, radiolabeled with the $\alpha$-emitter ${ }^{211} \mathrm{At}$.

The excised biologic samples were snap-frozen and cryosectioned as previously described (12). Ten to 20 pairs of consecutive sections were analyzed for each sample: one section for $\alpha$-camera imaging and the other section for staining with hematoxylin and eosin (H\&E) (in some cases, 3 consecutive sections were analyzed [Fig. 2]). The $\alpha$-camera image of radioactivity and the digitalized image of the H\&E section were manually coregistered with Adobe Photoshop CS5 software. A region of interest (ROI) was manually drawn on the $\alpha$-camera image with the ImageJ software (version 1.6.0_29; National Institutes of Health), as shown in Figure 1. The total pixel intensity within the ROI was quantified. A calibration curve was used to derive a coefficient that related the pixel intensity value to the activity of the $\alpha$-emitter, and this was used to estimate the activity in each ROI. This value was corrected for decay as discussed below (Eq. 4).

Similarly, an ROI was drawn on the digitalized H\&E section to evaluate the number of cells that corresponded to the measured activity. The number of cells in an ROI was estimated with the following equation:

$$
N b_{\text {Cells }, R O I}=S_{R O I} \times \Delta e \times P / V_{\text {Cell }}
$$

where $S_{R O I}$ is the ROI area, $\Delta e$ represents the tissue section thickness $(12 \mu \mathrm{m}), P$ represents the cell packing within micrometastases $(P=1$ was assumed), and $V_{\text {cell }}$ is the cell volume calculated as:

$$
V_{\text {Cell }}=\frac{4}{3} \times \pi \times R_{\text {cell }}^{3},
$$

where $R_{\text {cell }}$ stands for the cell radius. The distribution of apparent radii of isolated OVCAR-3 cells on the H\&E images was used to determine mean radius. The maximal value in this distribution was used as the mean radius $\left(R_{\text {cell }}\right)$.

The activity per cell ( $A_{\text {cell }}$; at a given time after injection) was derived by dividing the activity measured in the ROI of the $\alpha$-camera

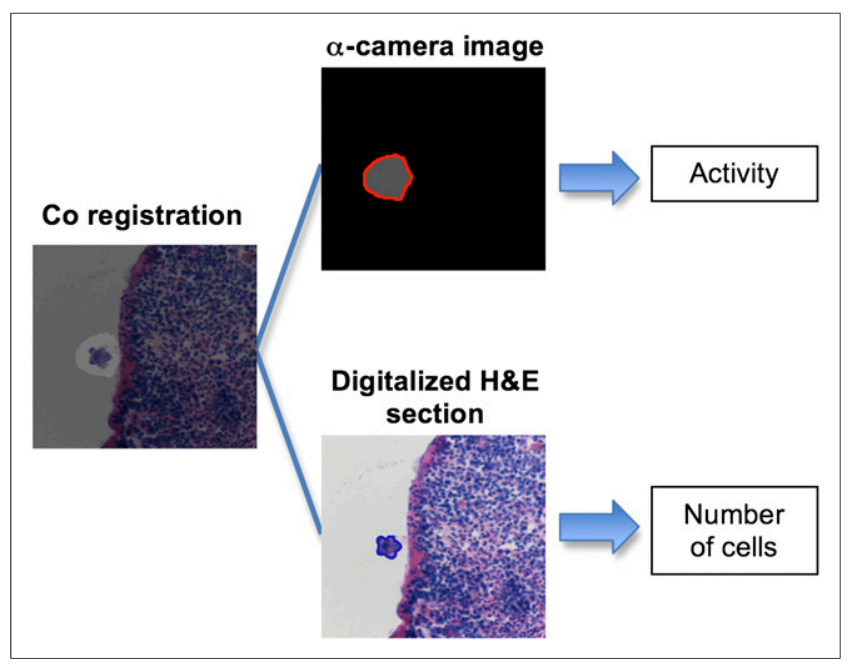

FIGURE 1. Illustration of different steps involved in activity quantification process.

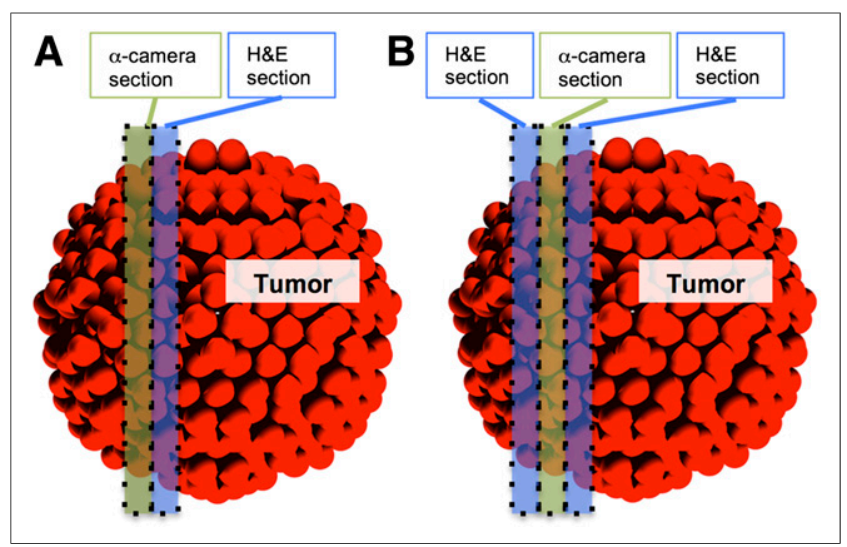

FIGURE 2. Description of sectioning scheme performed on biologic samples. (A) Method for all biologic samples: first section was used for $\alpha$-camera imaging and consecutive section for H\&E staining. In some cases, 3 consecutive sections were taken (B) to evaluate accuracy of cell number determination.

image $\left(A_{R O I}\right)$ by the number of cells within this part of the micrometastasis, $N b_{\text {Cells, ROI: }}$

$$
A_{\text {cell }}=\frac{A_{\text {ROI }}}{N b_{\text {Cells }, \text { ROI }}}=\frac{P I_{\text {ROI }} \times \text { Coeff }_{\text {Calib }} / \frac{1-e^{-d}}{d}}{S_{R O I} \times \Delta e \times P / V_{\text {Cell }}} \text { where } d=\lambda \times \Delta t,
$$

where $P I_{R O I}$ stands for the total pixel intensity in the defined ROI on the $\alpha$-camera image, Coeff Calib $_{\text {is }}$ is coefficient that transforms the pixel intensity into activity, $\Delta t$ is the acquisition time, and $\lambda$ is the decay constant of the $\alpha$-emitter.

\section{$\alpha$-Camera Images}

Three $\alpha$-camera systems were set up in parallel. The $\alpha$-camera system consisted of a cooled, high-sensitivity charge-coupled device (SXV-H9; Starlight Xpress Ltd.), coupled to a photographic lens (60 $\mathrm{mm}$ f/2.8 Micro-Nikkor; Nikon Corp.) (12). Specimens to be imaged (i.e., tissue sections on a scintillating sheet) were placed on an adjustable stand below the photographic lens. The distance of the specimen to the lens was adjusted to capture the entire specimen within the field of view.

A series of 600 - or 1,200-s images $(\sim 10)$ was acquired for each group of specimens. Postprocessing was performed as described previously (12).

The coefficient for converting the pixel intensity in an $\alpha$-camera image into activity was determined during the experiments. This coefficient depended on the specimen-to-lens distance. To establish the calibration curve for each camera, each sample was imaged with the $\alpha$-camera at a given pixel resolution (proportional to the specimen-tolens distance), then measured with a $\gamma$-counter (Wizard 1480; Wallac Oy). At each distance, and for numerous samples, the calibration coefficients were calculated by dividing the $\gamma$-counter-measured activity $(\mathrm{Bq})$ by the total pixel intensity per second of acquisition (pixel intensity unit $\cdot \mathrm{s}^{-1}$ ) for the whole tissue section. The calibration curves comprised the coefficient of calibration plotted as a function of the pixel resolution. Experimental points were fitted with a second-order polynomial curve.

Because the exposure times were long ( $\leq 2 \mathrm{~h}$ ), a correction was applied (Eq. 3 ) to the measured activity $\left(A_{\text {ROI,Meas }}\right)$ equal to $P I_{R O I} \times$ Coeff $f_{\text {Calib }}$ to correct for radioactive decay during the time of acquisition; that is, the activity was not constant during the acquisition period 
$(\Delta t)$. Using the decay constant of ${ }^{211} \mathrm{At}(\lambda)$, we derived the activity in an ROI at the start of the acquisition using the following equation:

$$
A_{R O I}=A_{R O I, \text { Meas }} / \frac{1-e^{-d}}{d} \quad \text { where } d=\lambda \times \Delta t .
$$

\section{Accuracy of Cell Number Determination}

The estimated cell number, from the H\&E section adjacent to the imaged $\alpha$-camera section, was used for deriving the activity per cell (Fig. 2A). The size of this introduced sampling error depended on the size and shape of the analyzed tumor cell cluster. To evaluate this error, a third section, from the other side of the $\alpha$-camera section (Fig. 2B), was evaluated. The number of cells in each identified cluster was determined in this third section $\left(N b_{\text {Cells,third }}\right)$. We considered the true cell number $\left(\mathrm{N} b_{\text {Cells.true }}\right)$ to be the average:

$$
N b_{\text {Cells }, \text { true }}=\frac{N b_{\text {Cells }, R O I}+N b_{\text {Cells }, 3 r d}}{2} .
$$

To estimate the uncertainty in the $N b_{C e l l s, R O I}$ value, the distributions of $\frac{N b_{\text {Cells,ROI }}}{N b_{\text {Cells,true }}}$ were characterized for different sizes of tumor cell clusters.

Other uncertainties of the method, including the general uncertainty, are detailed in the supplemental materials (available online only at http://jnm.snmjournals.org).

\section{Astatine}

The $\alpha$-emitter ${ }^{211}$ At was produced in a cyclotron (Scanditronix MC32; Cyclotron and PET Unit, Rigshospitalet) via the ${ }^{209} \mathrm{Bi}$ $(\alpha, 2 \mathrm{n})^{211} \mathrm{At}$ reaction and isolated with a dry distillation procedure (13). The half-life of ${ }^{211} \mathrm{At}$ is $7.2 \mathrm{~h}$, and the emitted $\alpha$-particles (7.45 and $5.87 \mathrm{MeV}$, with ranges in tissue of 70 and $48 \mu \mathrm{m}$ ) were generated through 2 branches of decay (58\% and $42 \%$, respectively).

\section{mAb}

The murine IgG1 class mAb MX35 $\left(\mathrm{F}\left(\mathrm{ab}^{\prime}\right)_{2}\right.$ fragments) targets the $\mathrm{NaPi} 2 \mathrm{~b}$ cell surface glycoprotein expressed on more than $90 \%$ of human epithelial ovarian cancers $(14,15)$.

\section{Cells}

The human ovarian cancer cell line NIH:OVCAR-3 (16) was obtained from the American Type Culture Collection. Cell cultures were maintained at the Department of Oncology at Sahlgrenska University Hospital.

\section{Antibody Labeling}

The mAb MX35 was labeled with ${ }^{211}$ At using the reagent m-MeATE ( $N$-succinimidyl 3-(trimethylstannyl)benzoate), as previously described (17). The specific activity achieved was $650 \mathrm{MBq} / \mathrm{mg}$ (i.e., 1 of $250 \mathrm{mAbs}$ labeled with an ${ }^{211} \mathrm{At}$ atom) for all the experiments. The radiochemical purity was more than $99 \%$, determined by methanol precipitation, and the immunoreactivity was about $85 \%$.

\section{Animals and Study Groups}

Female, nude BALB/c nu/nu mice ( $n=44$; age range, $5-7$ wk [Charles River Laboratories]) were inoculated intraperitoneally with $1 \times 10^{7} \mathrm{NIH}$ :OVCAR-3 cells in $0.2 \mathrm{~mL}$ of cell medium. After $3-8 \mathrm{wk}$ (to obtain micrometastases of variable sizes), the mice were intraperitoneally treated with $6 \mathrm{MBq}(5.8-7 \mathrm{MBq})$ or $1 \mathrm{MBq}(0.8-1.1 \mathrm{MBq})$ of ${ }^{211}$ At-MX35 $\mathrm{F}\left(\mathrm{ab}^{\prime}\right)_{2}$ in $0.75 \mathrm{~mL}$ of phosphate-buffered saline. For each activity ( 1 and $6 \mathrm{MBq})$, animals were sacrificed at $1.5 \mathrm{~h}(n=6+6)$, $4 \mathrm{~h}(n=6+6), 8 \mathrm{~h}(n=6+6)$, and $24 \mathrm{~h}(n=4+4)$. The spleen, stomach, liver, peritoneal wall samples, and suspected lymph nodes were excised and snap-frozen. All animal experiments were approved by the ethics committee of the University of Gothenburg.

\section{Time-Activity Curve}

The activity per cell $\left(A_{\text {cell }}\right.$; Eq. 3$)$ was derived by quantification of activity in hundreds of tumor cell clusters at 4 different time points after injection. $A_{\text {cell }}$ values were sorted into groups according to the size of the cluster, as measured on the digitalized H\&E sections. Two cluster sizes were considered: those with radii less than $13.5 \mu \mathrm{m}$ (corresponding to $<5$ cells in a $12-\mu \mathrm{m}$-thick section) and those with radii between 23.4 and $42.8 \mu \mathrm{m}$ (between 15 and 50 cells in a 12- $\mu \mathrm{m}$ thick section). The $A_{\text {cell }}$ versus time point curve (i.e., time-activity plot) for each group was fitted using the equation $y=(1-\exp (-a \times$ $t)) \times \exp (-b \times t)$. The time-integrated activity per cell was derived as the area under the fitting curve.

\section{Dosimetry}

Dosimetric calculations at the cellular level were performed to estimate the mean absorbed dose delivered to tumor cells for each injected activity ( 1 or $6 \mathrm{MBq}$ ). We used a validated code, based on analytic calculations, that is able to determine the mean absorbed doses to isolated cells or cells within spheroids, exposed to $\alpha$-particles emitted from variable compartments (cell surface, cytoplasm, nucleus, extracellular domain) (18). In the case of spheroids, the contributions both from radiolabeled antibodies on the cell and from cross-fire are used to calculate the mean absorbed dose. The time-integrated activity derived above was used as an input for the algorithm. Cells and nuclei were modeled as 2 concentric homogeneous spheres of density $1 \mathrm{~g} / \mathrm{cm}^{3}$ with radii of 6.9 and $5.5 \mu \mathrm{m}$, respectively. We calculated the mean absorbed dose delivered to isolated cells, based on the time-integrated activity determined for clusters with a $13.5-\mu \mathrm{m}$ radius, and the mean absorbed dose delivered to cells within a micrometastasis with a radius of $42.8 \mu \mathrm{m}$ (modeled by a spheroid containing 200 cells), based on the number of decays per cell (which was measured experimentally for this cluster size). The spatial resolution $(\sim 35 \mu \mathrm{m})$ of the $\alpha$-camera images in this study did not allow for discrimination of differences in cell uptake of the radiolabeled antibody according to position in the cluster, but no cold spots were observed within identified tumor cell clusters, therefore a uniform activity distribution was assumed.

\section{Statistical Analysis}

Mean values of activity per cell were compared using a 2-sample $t$ test with the PRISM 5 software (GraphPad Software, Inc.). $P$ values of less than 0.05 were considered significant. Data are expressed as mean $\pm \mathrm{SD}$.

\section{RESULTS}

\section{$\alpha$-Camera Calibration Coefficient}

The calibration coefficient for the $\alpha$-camera (Fig. 3) was derived by plotting the measured activity $(\mathrm{Bq})$ divided by the pixel intensity per second of acquisition as a function of the relative pixel size $(\mu \mathrm{m})$ in the field of view (proportional to the specimento-lens distance). The experimental data were satisfactorily fitted with a second-order polynomial curve: $y=b_{0}+b_{1} \times x+b_{2} \times x^{\wedge} 2$ $\left(b_{0}=3.973 \times 10^{-3} \pm 9.07 \times 10^{-4} ; b_{1}=-3.32 \times 10^{-4} \pm 1.14 \times\right.$ $\left.10^{-4} ; b_{2}=1.93 \times 10^{-5} \pm 3.3 \times 10^{-6}\right)$.

\section{Accuracy of Number of Cells Determination}

Figure 4 shows the distributions of $\frac{N b_{\text {Cells,ROI }}}{N b_{\text {Cells, true }}}$ for 2 sizes of clusters $(<13.5-\mu \mathrm{m}$ radius or $23.4-$ to $42.8-\mu \mathrm{m}$ radius). The clusters with large radii showed a gaussian distribution, with a mean value of 0.96 and an SD of 0.26 , indicating a relatively good 


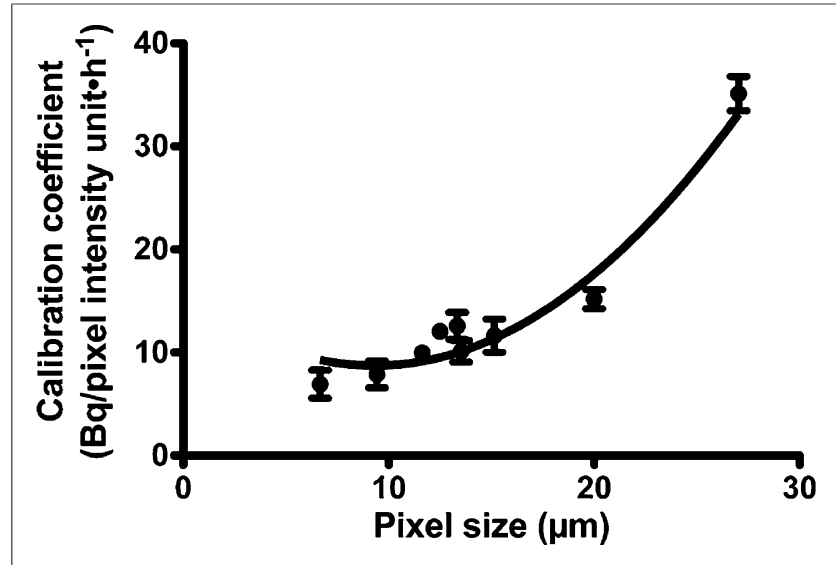

FIGURE 3. Coefficient of calibration (that relates activity to pixel intensity on $\alpha$-camera images per second of acquisition) as function of pixel size on $\alpha$-camera image for $1 \alpha$-camera. Experimental points were fitted with second-degree polynomial curve.

accuracy of the cell number determination for large clusters. The small clusters did not show a gaussian distribution. In a significant number of cases ( $41 \%$ of the analyzed clusters), the number of cells used to quantify the activity per cell $\left(\mathrm{Nb} b_{\text {Cells, ROI }}\right)$ was twice the number of cells estimated from 3 consecutive sections $\left(N b_{\text {Cells,true }}\right)$. This result indicated a systematic error. A ratio of 2 reflected the case when no cells were observed in 1 of the $2 \mathrm{H} \& \mathrm{E}$ sections.

\section{Experimental Measurements}

At different time points, the animals were dissected and spleen and stomach were excised. A significant number of tumor clusters $(>30)$ were typically found in one third of the animals at $1.5,4$, and $8 \mathrm{~h}$ for both injected activities. At $24 \mathrm{~h}$, only one 6-MBq animal could be used for activity uptake measurements. For $1-\mathrm{MBq}$ animals, the $\alpha$-camera image signals were too low for analysis at the 24-h time point (i.e., the delineation of tumor ROIs was impossible due to a low signal-to-noise ratio).

Figure 5 shows examples of coregistration of $\alpha$-camera images and the digitalized adjacent $\mathrm{H} \& \mathrm{E}$ section, displaying numerous tumor cell clusters by the peritoneal surface of the spleen. The activity distribution from the $\alpha$-camera image closely matched the anatomic features. $\alpha$-camera images were acquired from samples of different animals, and one can notice micrometastases of variable sizes.

Figure $6 \mathrm{~A}$ represents the time-activity plots (decay-corrected) for tumor clusters with less than $13.5-\mu \mathrm{m}$ radii for both injected activities (1 and $6 \mathrm{MBq}$ ). The small tumor clusters represented a situation similar to an isolated cell. For the $6-\mathrm{MBq}$ injection, at $1.5 \mathrm{~h}$, we observed a maximal uptake of $\approx 0.07 \pm 0.02 \mathrm{~Bq} /$ cell (corresponding to 2,100 radiolabeled antibodies per cell). After the $1-\mathrm{MBq}$ injection, the maximal uptake was $\approx 0.02 \pm 0.01$ $\mathrm{Bq} / \mathrm{cell}$ (i.e., 700 radiolabeled antibodies per cell). For both injections, up to $8 \mathrm{~h}$, the number of radiolabeled antibodies attached to tumor cells remained nearly constant (regardless of physical decay). At $24 \mathrm{~h}$ after the 6-MBq injection, the cluster activity uptake had decreased from 2,100 to around 800 radiolabeled antibodies per cell. Both experimental plots (for 1 and $6 \mathrm{MBq}$ ) were fitted with a standard antibody binding equation (assuming antibody excess in the peritoneal cavity) applied with PRISM 5 software:

$$
\begin{aligned}
& A_{\text {Cell }}=B_{\text {max }} \times\left(\frac{S A \times M}{N_{A}}\right) \times \frac{K_{o n} \times r \times\left(\frac{A_{i n j}}{S A \times M \times V_{i n j}}\right)}{K_{o n} \times r \times\left(\frac{A_{i n j}}{S A \times M \times V_{i n j}}\right)+K_{o f f}} \times \\
& \left(1-\exp \left(-\left(K_{o n} \times r \times\left(\frac{A_{i n j}}{S A \times M \times V_{i n j}}\right)+K_{o f f}\right) \times t\right)\right)
\end{aligned}
$$

Here, $B_{\max }$ represents the total number of antigens on the tumor cell surface, $S A$ is the specific activity of the ${ }^{211}$ At-MX35 antibody (650 MBq/mg), $M$ is the molar mass of MX35 F(ab') $2(100,000 \mathrm{~g} /$ $\mathrm{mol}), N_{A}$ is the Avogadro constant, $r$ is the immunoreactive fraction (0.82), $A_{i n j}$ is the injected activity ( 6 or $1 \mathrm{MBq}$ ), and $V_{i n j}$ is the injected solution volume $(0.75 \mathrm{~mL})$. The fits of both curves provided a $B_{\text {max }}$ of $7 \times 10^{5} \pm 1.8 \times 10^{5}$, the $K_{\text {on }}$ was $44,000 \mathrm{~L} \mathrm{~mol}^{-1}$ $\mathrm{s}^{-1}$, and the $K_{\text {off }}$ was $2.2 \times 10^{-3} \mathrm{~s}^{-1} \pm 0.67 \times 10^{-3}$. The value of $K_{o n}$ was fixed as a constant in the fitting equation, based on previous in vitro measurements (19).

Figure 6B represents the time-activity plots (decay-corrected) for tumor clusters with 23.4- to $42.8-\mu \mathrm{m}$ radii for both injected activities (1 and $6 \mathrm{MBq})$. The curve shapes were similar for large
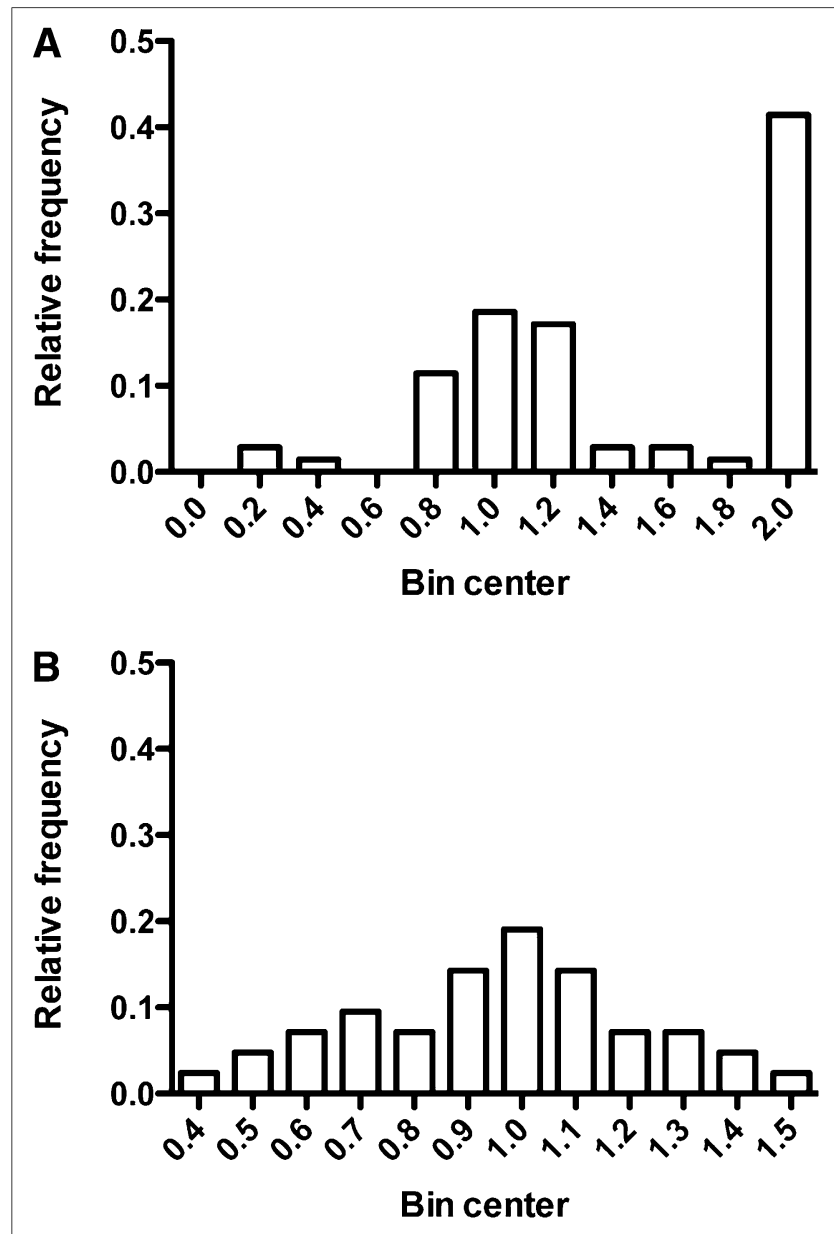

FIGURE 4. Distributions of ratio $\frac{N b_{\text {Cells,ROI }}}{N b_{\text {Cells.true }}}$ (i.e., number of cells estimated by general method divided by number of cells estimated by use of 3 consecutive sections) for 2 sizes of clusters $(<13.5-\mu \mathrm{m}$ radius $[\mathrm{A}]$ and $23.4-$ to $42.8-\mu \mathrm{m}$ radius $[\mathrm{B}])$. 


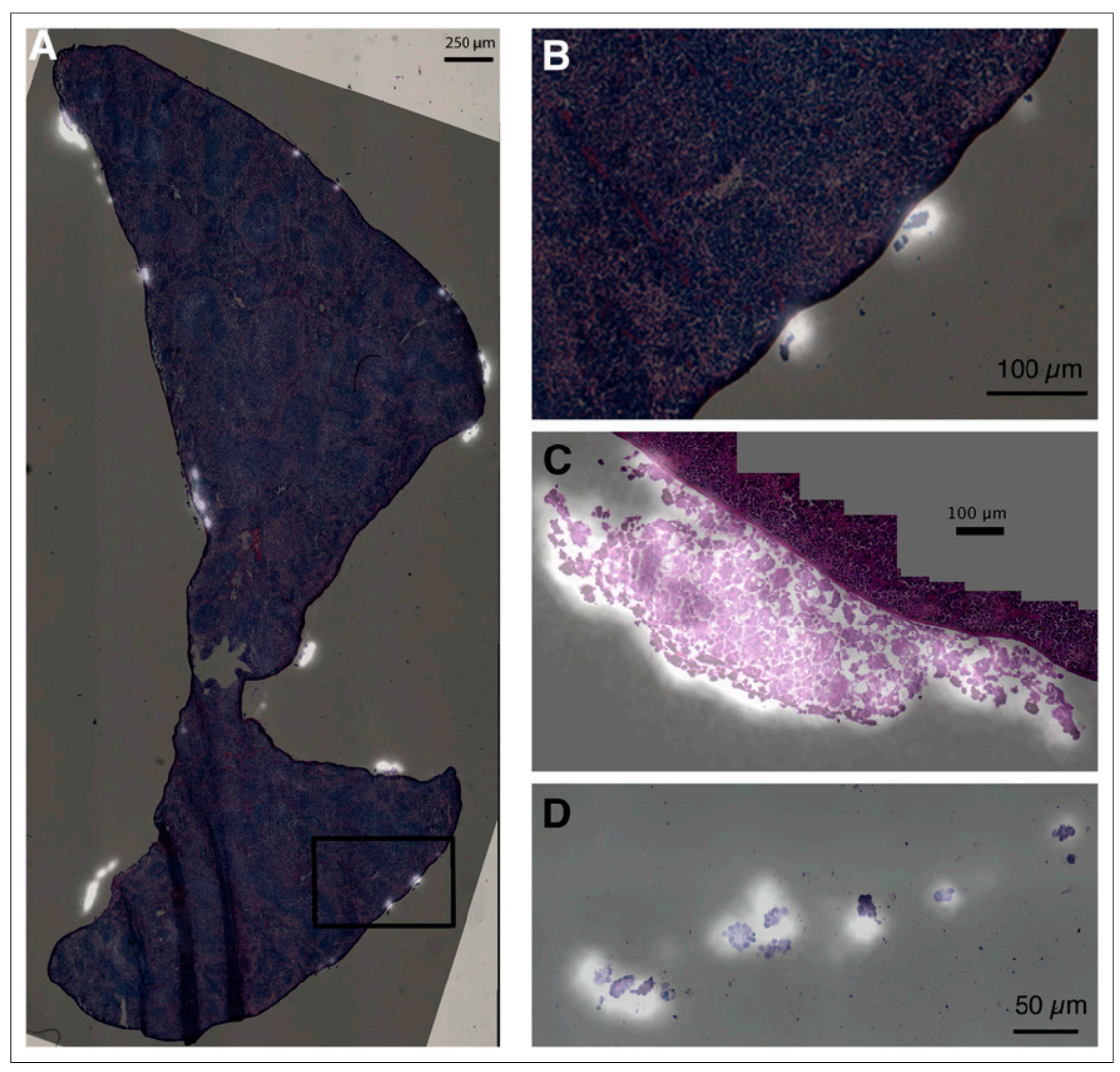

FIGURE 5. Coregistration of $\alpha$-camera images and their consecutive H\&E section. Bright areas represent areas of high activity. (A) Whole section of spleen with numerous tumor cell clusters for which activity uptake is significant $4 \mathrm{~h}$ after injection. (B) Blow up of previous image showing 4 clusters and corresponding activity spots on $\alpha$-camera image. (C) Nonvascularized micrometastasis $(400-\mu \mathrm{m}$ thick and $800-\mu \mathrm{m}$ wide) attached to spleen. Eight hours after injection, activity distribution appeared slightly uniform. (D) Detail of section of drop of ascitic fluid containing tumor cell clusters.

and small clusters, but the uptake per cell was significantly lower for large clusters (Fig. 6B). For 6-MBq injections, the activity per cell was constant from $1.5 \mathrm{~h}(\approx 0.027 \pm 0.010 \mathrm{~Bq} /$ cell $)$ to $4 \mathrm{~h}(\approx 0.034 \pm$ $0.012 \mathrm{~Bq} / \mathrm{cell})$, then decreased slightly at $8 \mathrm{~h}(\approx 0.015 \pm 0.010 \mathrm{~Bq} /$ cell). For $1-\mathrm{MBq}$ injections, the maximum uptake was observed around $1.5 \mathrm{~h}(\approx 0.005 \pm 0.002 \mathrm{~Bq} / \mathrm{cell})$ and decreased thereafter.

The time-integrated activity for small tumor clusters was over 1,700 decays per cell for a $6-\mathrm{MBq}$ injection, as derived from the lines shown in Figure 6A. This value decreased to 500 decays per cell for a $1-\mathrm{MBq}$ injection. Using these values, we estimated the mean absorbed dose delivered to isolated cells, and they were approximately 40 and 12 Gy for injections of 6 and $1 \mathrm{MBq}$, respectively. For the large tumor clusters, around 750 and 180 decays per cell were detected for the 6- and $1-\mathrm{MBq}$ injections, respectively. Assuming a uniform activity distribution in those clusters, the calculations showed that cells received between 125 and $245 \mathrm{~Gy}$ for the 6-MBq injections and between 30 and $60 \mathrm{~Gy}$ for the $1-\mathrm{MBq}$ injections (with $>85 \%$ of the mean absorbed dose originating from cross-fire irradiation).

\section{DISCUSSION}

Because of the characteristics of $\alpha$-particles, TAT is expected to be a potent treatment for circulating, minimal, and residual malignancies. The development of radioimmunoconjugates for those indications involves complex evaluations. Consequently, many evaluations rely on in vitro measurements of radiolabeled vector binding and tumor cell survival (20) or on the estimation of in vivo efficacy for different kinds of malignant lesions (e.g., subcutaneous tumors grafted on mice) (21). For ovarian carcinoma, TATs were evaluated with a model mimicking the human minimal disease (i.e., peritoneal spread of micrometastases) to determine the efficacy of ${ }^{211}$ At-MX35 and compare it with other radiolabeled specific (19) and nonspecific antibodies (22). The endpoint was the fraction of animals that presented no visible signs of tumor at $8 \mathrm{wk}$ after therapy. These studies contributed to the initiation of a clinical trial (7); however, numerous questions remained unanswered and prevented proper optimization. In general, it has been difficult to measure pre- and posttherapy tumor statuses; thus, the efficacy evaluation can be misleading (23).

We demonstrate here a method using ex vivo measurements to assess activity uptake in micrometastases and even in isolated tumor cells. We showed that the time-integrated activity could be measured directly in targeted tumor clusters or tumor cells, enabling comparisons of the in vivo action of different radioimmunoconjugate regimens. Previous attempts to measure activity uptake on micrometric tumor structures with autoradiography have been confined to snapshot measurements that provided the activity distribution at only 1 time point after treatment injection $(24,25)$. The present method showed that the $\alpha$-camera could be used to determine both spatial and temporal activity distributions, despite the need to analyze samples from different sacrificed animals.

\section{Uncertainties}

To validate this technique and evaluate its accuracy, we detailed and estimated the uncertainties associated with each step of the calculation (supplemental materials). The global relative uncertainty on the value of activity per cell was estimated to be approximately $75 \%$ for a case example of a tumor cluster of 42.8 $\mu \mathrm{m}$ in radius. We expected that an analysis of $10-15$ clusters would be sufficient to achieve an acceptable confidence level (i.e., a relative uncertainty close to $20 \%$ ). This process highlighted difficulties in determining the number of cells in very small tumor cell clusters $(<13.5 \mu \mathrm{m}$ in radius $)$ - that is, close to 1 cell. The distribution of the ratio (measured value:true estimation of the value) was non-gaussian, indicating a potential systematic error. This error was not observed for large clusters. To investigate the impact of this error, we created another time-activity curve based on $2 \mathrm{H} \& \mathrm{E}$-stained sections to determine the number of cells. We analyzed several clusters at different time points $(n=20+25$ at $1.5 \mathrm{~h}, n=30$ at $4 \mathrm{~h}$, and $n=9$ at $8 \mathrm{~h}$ ). As expected, the activity per cell determined by this method was slightly higher when based on 3, rather than 2, adjacent sections. This difference was not significant, given the error bars. For example, from the 2 animals 


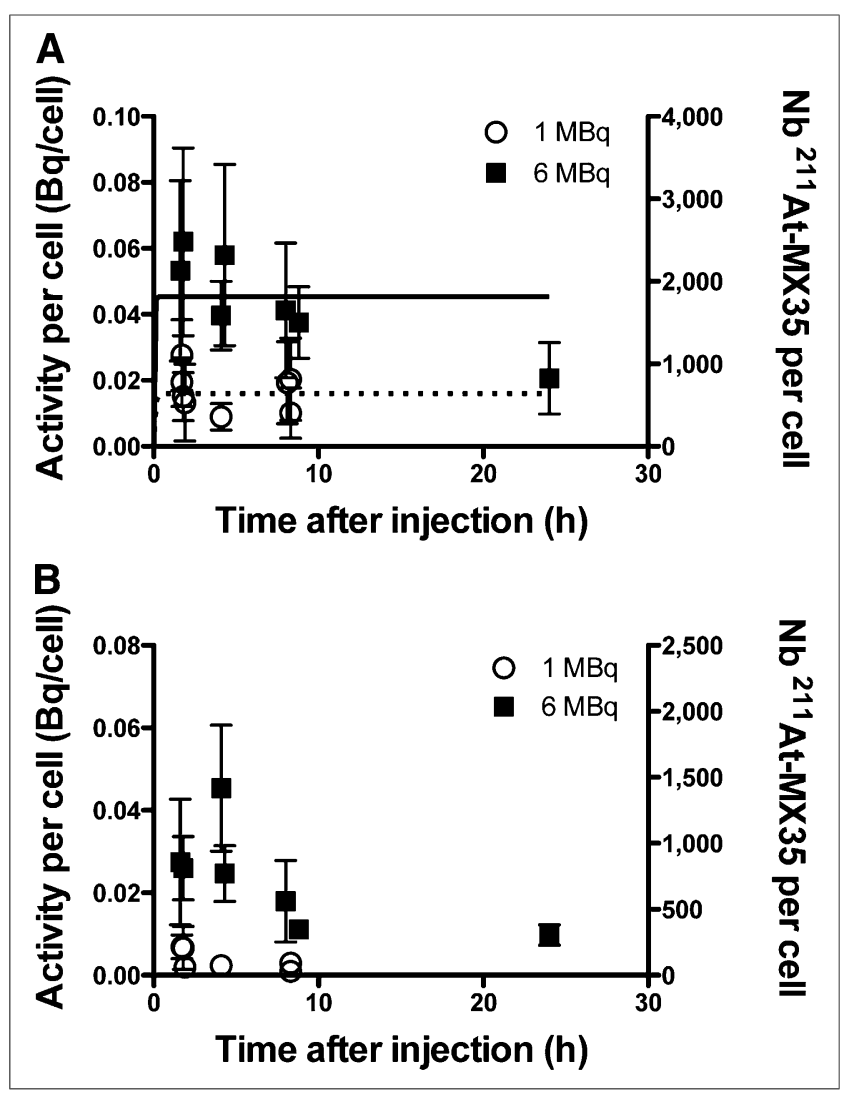

FIGURE 6. Activity per cell (left $y$-axis) and number of radiolabeled antibodies per cell (right $y$-axis) as function of time after injection for 2 different injected activities (1 and $6 \mathrm{MBq}$ ) within micrometastases less than $13.5 \mu \mathrm{m}$ in radius $(\mathrm{A})$ and within micrometastases comprised between 23.4 and $42.8 \mu \mathrm{m}$ (B). Experimental points were decay-corrected. Error bars represent SD of all experimental measurements at each time point. For micrometastases less than 13.5 $\mu \mathrm{m}$ in radius (A), plots for both activities were fitted (plain line for 6 $\mathrm{MBq}$, dotted line for $1 \mathrm{MBq}$ ) with classic antibody binding equation (Eq. 6). Value at $24 \mathrm{~h}$ and for $6 \mathrm{MBq}$ was discarded in fitting process.

studied at $1.5 \mathrm{~h}$, the activities were $0.044 \pm 0.021$ and $0.059 \pm$ $0.028 \mathrm{~Bq} /$ cell based on 3 sections and $0.040 \pm 0.018$ and $0.052 \pm$ $0.028 \mathrm{~Bq} /$ cell based on 2 sections. Nevertheless, we recommend that future studies on small biologic structures should be based on 3 consecutive sections to increase confidence in the results.

Experimental values for the largest analyzed clusters were associated with a relative SD between $15 \%$ and $50 \%$, where around 10 clusters were analyzed at each time point. This SD was, in some cases, slightly higher than theoretic estimations. However, previous studies observed relatively high biologic variability in the number of antigens present at the surface of tumor cells $(26,27)$, possibly explaining the discrepancy between our theoretic estimations and experimental measurements.

\section{Time-Activity Curves}

In the murine model used in this work, we targeted micrometastases in the peritoneal cavity with intraperitoneal injections of the ${ }^{211}$ At-MX35 antibody at 2 different injected activities. In previous studies, activity uptake by micrometastases was modeled with binding parameters determined in vitro (19). Because of the micrometric nature of the malignant lesions, no previous studies have performed in vivo assessments of tumor uptake. With the present method, it was possible to make experimentally based measurements and create a time-activity curve for micrometastases as small as a few tumor cells. Using this data, we derived relevant values of the mean absorbed dose delivered to tumor cells. We found good radiolabel stability for up to $8 \mathrm{~h}$, with a constant number of antibodies on the surfaces of cells. At $24 \mathrm{~h}$, and to a lesser degree at $8 \mathrm{~h}$, the measured activity per cell declined. It was not possible to ascertain whether this decline resulted from instability of the antigen-antibody association, from conjugate instability (less probable according to in vitro results demonstrating good stability of the labeling method (17)), or from a measurement artifact (at late time points, the signal-to-noise ratio had declined on the $\alpha$-camera images; thus, data were less reliable). Additionally, cell damage after 8-24 h of acute irradiation could induce measurement errors. We assume that ultrastructural changes in irradiated cells, receiving more than $10 \mathrm{~Gy}$ of $\alpha$-particle irradiation, could affect the results derived from our method.

\section{Small-Scale Dosimetry}

When time-activity curves for 6- and $1-\mathrm{MBq}$ injections were fitted, we found in vivo values of $B_{\max }\left(7 \times 10^{5}\right)$ and antibody dissociation constant, $K_{d}\left(5 \times 10^{-8} \mathrm{M}\right)$. Previously (19), in vitro measurements indicated that OVCAR-3 cells possessed approximately $1 \times 10^{6}$ antigens, and the antibody $K_{\text {off }}$ was negligible. In the present work, the maximal uptake on tumor cells depended on the injected activity. This could argue for the possibility of antibody dissociation. From previous in vitro measurements, theoretic modeling estimated that the number of decays per cell would be more than 800 for an injected activity of $1 \mathrm{MBq}$, taking into account a specific activity of $134 \mathrm{MBq} / \mathrm{mg}$, which was used at that time (19). Those values were previously used for small-scale dosimetry and the establishment of dose-effect curves. However, the 2 dose-effect curves reported at that time for injections of nonspecific and specific radiolabeled antibodies were different, possibly indicating an overestimation of the mean absorbed dose delivered in the case of specific antibodies (22). In the present study, the measured time-integrated activity was around 500 decays per cell for a $1-\mathrm{MBq}$ injection (specific activity, $650 \mathrm{MBq} / \mathrm{mg}$, or 4.8 times the specific activity used in previous studies). This value was 7.7 times less than the theoretically modeled value (scaling for the specific activity). On the basis of our experimental time-integrated activity, we calculated that the mean self-absorbed dose delivered to OVCAR-3 cells was around $12 \mathrm{~Gy}$, a highly therapeutic dose, because a $\mathrm{D}_{0}$ (the mean absorbed dose required to reduce cell survival to $37 \%$ ) of 0.56 Gy was determined in vitro for OVCAR-3 cells (9).

Interestingly, clusters with radii greater than $20 \mu \mathrm{m}$ had significantly lower uptake than smaller clusters, regardless of the injected activity. At $1.5 \mathrm{~h}$, after a $1-\mathrm{MBq}$ injection, the activity was less than $0.025 \mathrm{~Bq} /$ cell for large micrometastases and around $0.05 \mathrm{~Bq} /$ cell for an isolated tumor cell $(P=0.018)$.

For large tumor clusters $(>100 \mu \mathrm{m}$ in diameter), slow penetration of radiolabeled antibodies through the tumors was reported (28-30). We therefore expected that the number of radiolabeled antibodies per tumor cell (after decay-correction) would increase with time. For both injected activities, this was not observed in the first $8 \mathrm{~h}$ after the injection. Within large micrometastases $(>500$ $\mu \mathrm{m})$ containing more than 5,000 cells, the activity distribution showed no ring-effect - that is, presence of activity only at the edge of the micrometastasis-at early time points (Fig. 5C). In contrast, the distribution appeared uniform. 
Despite the lower uptake per cell in the large clusters, the timeintegrated activity was still high; an average of 500 decays per cell was measured within micrometastases of $23.4-42.8 \mu \mathrm{m}$ in radii (i.e., between 15 and 50 cells in a $12-\mu \mathrm{m}$-thick section) for $1-\mathrm{MBq}$ injections. Although activity distribution was unknown in micrometastases, the range of possible mean absorbed doses could be estimated by considering 2 opposing scenarios: a uniform distribution of activity or a skewed distribution. In the latter scenario, all the labeled antibodies (500 decays per cell $\times$ number of cells in the micrometastasis) were assumed to be attached to tumor cells at the edge of the lesion. When a uniform distribution of decays was assumed within a large micrometastasis $(42.8-\mu \mathrm{m}$ radius), the mean absorbed dose delivered to cells was between 30 (cells on the edge) and $60 \mathrm{~Gy}$ (cells in the core). When a skewed distribution of decays was assumed for the micrometastasis, the lowest mean absorbed dose in the tumor was 26 Gy. Again, those values are expected to be therapeutic, and they may explain the good therapeutic outcomes previously observed (22). Further studies on penetration of MX35 within intraperitoneal larger metastases are ongoing.

\section{CONCLUSION}

The present work demonstrated a method for measuring timeactivity curves at the cellular scale from experimental data. Our results provided information on the binding of a specific radiolabeled antibody to ovarian carcinoma micrometastases. We measured a high activity uptake, particularly for micrometastases of only a few tumor cells. This quantitative method represents a step forward in the development of small-scale dosimetry, because further modeling was not required to derive the spatial and temporal activity distributions in micrometric biologic structures. This method is not limited to preclinical experiments; in future, it could be applied to the analysis of tumor material excised from patients undergoing surgery.

\section{DISCLOSURE}

The costs of publication of this article were defrayed in part by the payment of page charges. Therefore, and solely to indicate this fact, this article is hereby marked "advertisement" in accordance with 18 USC section 1734. This work was supported by grants from the Swedish Cancer Society, the Swedish Research Council, the Swedish Radiation Safety Authority, the Assar Gabrielsson Foundation, and the King Gustaf V Jubilee Clinic Research Foundation in Gothenburg, Sweden. No other potential conflict of interest relevant to this article was reported.

\section{REFERENCES}

1. Macklis RM, Kinsey BM, Kassis AI, et al. Radioimmunotherapy with alphaparticle-emitting immunoconjugates. Science. 1988;240:1024-1026.

2. Sgouros G. Alpha-particles for targeted therapy. Adv Drug Deliv Rev. 2008;60:1402-1406.

3. Mulford DA, Scheinberg DA, Jurcic JG. The promise of targeted $\alpha$-particle therapy. J Nucl Med. 2005;46(suppl 1):199S-204S.

4. Chérel M, Davodeau F, Kraeber-Bodere F, Chatal JF. Current status and perspectives in alpha radioimmunotherapy. Q J Nucl Med Mol Imaging. 2006;50:322-329.

5. Jurcic JG, Larson SM, Sgouros G, et al. Targeted alpha particle immunotherapy for myeloid leukemia. Blood. 2002;100:1233-1239.

6. Rosenblat TL, McDevitt MR, Mulford DA, et al. Sequential cytarabine and alpha-particle immunotherapy with bismuth-213-lintuzumab (HuM195) for acute myeloid leukemia. Clin Cancer Res. 2010;16:5303-5311.

7. Andersson H, Cederkrantz E, Back T, et al. Intraperitoneal alpha-particle radioimmunotherapy of ovarian cancer patients: pharmacokinetics and dosimetry of ${ }^{211}$ At-MX35 F(ab')2-a phase I study. J Nucl Med. 2009;50:1153-1160.
8. Zalutsky MR, Reardon DA, Akabani G, et al. Clinical experience with alphaparticle emitting ${ }^{211} \mathrm{At}$ : treatment of recurrent brain tumor patients with ${ }^{211} \mathrm{At}-$ labeled chimeric antitenascin monoclonal antibody 81C6. J Nucl Med. 2008;49:30-38.

9. Palm S, Andersson H, Back T, et al. In vitro effects of free ${ }^{211} \mathrm{At},{ }^{211}$ At-albumin and ${ }^{211}$ At-monoclonal antibody compared to external photon irradiation on two human cancer cell lines. Anticancer Res. 2000;20:1005-1012.

10. Miederer M, Seidl C, Beyer GJ, et al. Comparison of the radiotoxicity of two alpha-particle-emitting immunoconjugates, terbium-149 and bismuth-213, directed against a tumor-specific, exon 9 deleted (d9) E-cadherin adhesion protein. Radiat Res. 2003;159:612-620.

11. Dahle J, Krogh C, Melhus KB, Borrebaek J, Larsen RH, Kvinnsland Y. In vitro cytotoxicity of low-dose-rate radioimmunotherapy by the alpha-emitting radioimmunoconjugate thorium-227-DOTA-rituximab. Int J Radiat Oncol Biol Phys. 2009;75:886-895.

12. Bäck T, Jacobsson L. The alpha-camera: a quantitative digital autoradiography technique using a charge-coupled device for ex vivo high-resolution bioimaging of alpha-particles. J Nucl Med. 2010;51:1616-1623.

13. Lindegren S, Back T, Jensen HJ. Dry-distillation of astatine-211 from irradiated bismuth targets: a time-saving procedure with high recovery yields. Appl Radiat Isot. 2001;55:157-160.

14. Welshinger M, Yin BW, Lloyd KO. Initial immunochemical characterization of MX35 ovarian cancer antigen. Gynecol Oncol. 1997;67:188-192.

15. Mattes MJ, Look K, Furukawa K, et al. Mouse monoclonal antibodies to human epithelial differentiation antigens expressed on the surface of ovarian carcinoma ascites cells. Cancer Res. 1987;47:6741-6750.

16. Hamilton TC, Young RC, McKoy WM, et al. Characterization of a human ovarian carcinoma cell line (NIH:OVCAR-3) with androgen and estrogen receptors. Cancer Res. 1983;43:5379-5389.

17. Lindegren S, Frost S, Back T, Haglund E, Elgqvist J, Jensen H. Direct procedure for the production of ${ }^{211}$ At-labeled antibodies with an epsilon-lysyl-3-(trimethylstannyl)benzamide immunoconjugate. J Nucl Med. 2008;49:1537-1545.

18. Chouin N, Bernardeau K, Davodeau F, et al. Evidence of extranuclear cell sensitivity to alpha-particle radiation using a microdosimetric model. I. Presentation and validation of a microdosimetric model. Radiat Res. 2009;171:657663.

19. Elgqvist J, Andersson H, Back T, et al. Therapeutic efficacy and tumor dose estimations in radioimmunotherapy of intraperitoneally growing OVCAR-3 cells in nude mice with ${ }^{211}$ At-labeled monoclonal antibody MX35. J Nucl Med. 2005;46:1907-1915.

20. Nikula TK, McDevitt MR, Finn RD, et al. Alpha-emitting bismuth cyclohexylbenzyl DTPA constructs of recombinant humanized anti-CD33 antibodies: pharmacokinetics, bioactivity, toxicity and chemistry. J Nucl Med. 1999;40:166-176.

21. Zalutsky MR, Stabin MG, Larsen RH, Bigner DD. Tissue distribution and radiation dosimetry of astatine-211-labeled chimeric 81C6, an alpha-particle-emitting immunoconjugate. Nucl Med Biol. 1997;24:255-261.

22. Elgqvist J, Andersson H, Back T, et al. Alpha-radioimmunotherapy of intraperitoneally growing OVCAR-3 tumors of variable dimensions: outcome related to measured tumor size and mean absorbed dose. J Nucl Med. 2006;47:1342-1350.

23. Frost SHL, Bäck T, Chouin N, et al. Comparison of ${ }^{211}$ At-PRIT and ${ }^{211}$ At-RIT of ovarian microtumors in a nude mouse model. Cancer Biother Radiopharm. 2013; 28:108-114.

24. Akabani G, Kennel SJ, Zalutsky MR. Microdosimetric analysis of alpha-particleemitting targeted radiotherapeutics using histological images. J Nucl Med. 2003;44:792-805.

25. Lu XQ, Kiger WS. Application of a novel microdosimetry analysis and its radiobiological implication for high-LET radiation. Radiat Res. 2009;171:646656.

26. Kvinnsland Y, Stokke T, Aurlien E. Radioimmunotherapy with alpha-particle emitters: microdosimetry of cells with a heterogeneous antigen expression and with various diameters of cells and nuclei. Radiat Res. 2001;155:288-296.

27. Neti PV, Howell RW. Log normal distribution of cellular uptake of radioactivity: implications for biologic responses to radiopharmaceuticals. J Nucl Med. 2006;47:1049-1058.

28. Ito T, Griffin TW, Collins JA, Brill AB. Intratumoral and whole-body distributions of $\mathrm{C} 110$ anti-carcinoembryonic antigen radioimmunotoxin after intraperitoneal and intravenous injection: a quantitative autoradiographic study. Cancer Res. 1992;52:1961-1967.

29. Flessner MF, Choi J, Credit K, Deverkadra R, Henderson K. Resistance of tumor interstitial pressure to the penetration of intraperitoneally delivered antibodies into metastatic ovarian tumors. Clin Cancer Res. 2005;11:3117-3125.

30. Choi J, Credit K, Henderson K, et al. Intraperitoneal immunotherapy for metastatic ovarian carcinoma: resistance of intratumoral collagen to antibody penetration. Clin Cancer Res. 2006;12:1906-1912. 